\title{
Hypoxia and $\mathrm{H}_{2} \mathrm{O}_{2}$ Dual-Sensitive Vesicles for Enhanced Glucose- Responsive Insulin Delivery
}

\author{
Jicheng Yu, ${ }^{\dagger, \ddagger}$ Chenggen Qian, ${ }^{\dagger, \S}$ Yuqi Zhang, ${ }^{\dagger \dagger}$ Zheng Cui, ${ }^{\|}$Yong Zhu, ${ }^{\dagger, \|}$ Qundong Shen, ${ }^{\S}$ \\ Frances S. Ligler, John B. Buse, ${ }^{\perp}$ and Zhen $\mathrm{Gu}^{*, \dagger, \downarrow, \perp}$ \\ ${ }^{\dagger}$ Joint Department of Biomedical Engineering, University of North Carolina at Chapel Hill and North Carolina State University, \\ Raleigh, North Carolina 27695, United States \\ ${ }^{\ddagger}$ Center for Nanotechnology in Drug Delivery and Division of Molecular Pharmaceutics, UNC Eshelman School of Pharmacy, \\ University of North Carolina at Chapel Hill, Chapel Hill, North Carolina 27599, United States \\ ${ }^{\S}$ Department of Polymer Science \& Engineering and Key Laboratory of High Performance Polymer Materials \& Technology of MOE, \\ School of Chemistry \& Chemical Engineering, Nanjing University, Nanjing, 210023, China \\ "Department of Mechanical and Aerospace Engineering, North Carolina State University, Raleigh, North Carolina 27695, United \\ States \\ ${ }^{\perp}$ Department of Medicine, University of North Carolina at Chapel Hill, Chapel Hill, North Carolina 27599, United States
}

\begin{abstract}
A glucose-responsive closed-loop insulin delivery system mimicking pancreas activity without long-term side effect has the potential to improve diabetic patients' health and quality of life. Here, we developed a novel glucose-responsive insulin delivery device using a painless microneedle-array patch containing insulin-loaded vesicles. Formed by self-assembly of hypoxia and $\mathrm{H}_{2} \mathrm{O}_{2}$ dual-sensitive diblock copolymer, the glucose-responsive polymersomebased vesicles ( $d$-GRPs) can disassociate and subsequently release insulin triggered by $\mathrm{H}_{2} \mathrm{O}_{2}$ and hypoxia generated during glucose oxidation catalyzed by glucose specific enzyme. Moreover, the $d$-GRPs were able to eliminate the excess $\mathrm{H}_{2} \mathrm{O}_{2}$, which may lead to free radicalinduced damage to skin tissue during the long-term usage and reduce the activity of GOx. In vivo experiments indicated that this smart insulin patch could efficiently regulate the blood glucose in the chemically induced type 1 diabetic mice for $10 \mathrm{~h}$.
\end{abstract}

KEYWORDS: Drug delivery, diabetes, insulin, glucose-responsive, hypoxia-sensitive, $\mathrm{H}_{2} \mathrm{O}_{2}$-sensitive

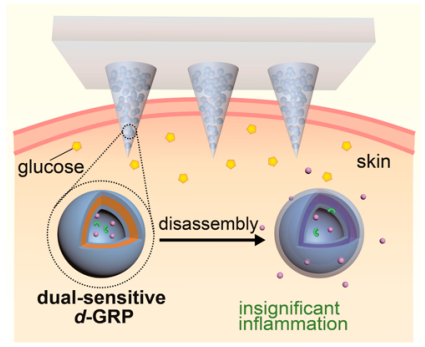

$\mathrm{D}$ iabetes mellitus is a chronic disease associated with elevated glucose in the blood, which currently affects 415 million people worldwide. ${ }^{1,2}$ Insulin, a hormone to help cells take in glucose for energy, is essential for the treatment of type 1 and advanced type 2 diabetic patients in order to maintain normoglycemia. ${ }^{3}$ However, the traditional exogenous insulin injection does not closely match the physiological release of insulin, often resulting in inadequate glycemic control ${ }^{2,4}$ and subsequent consequences such as limb amputation, blindness, and kidney failure. In addition, overtreatment with insulin may lead to hypoglycemia, which can cause behavioral and cognitive disturbance, seizures, loss of consciousness, brain damage, and even death. ${ }^{5}$ A "smart" glucose-responsive insulin delivery system that can mimic the $\beta$-cells to "secrete" insulin in response to a high blood glucose level (BGL) is desirable to regulate glycemia with minimal effort and to improve the health and quality of life for diabetic patients. ${ }^{1,2,6,7}$

In order to achieve this goal, such closed-loop systems usually consist of a glucose monitoring module and an insulin releasing module. ${ }^{1,2}$ The current closed-loop electromechanical systems include a continuous glucose sensor and an external insulin infusion pump. ${ }^{2}$ However, there are still some challenges that limit the application of these systems, such as lag in blood glucose feedback and biofouling. ${ }^{8}$ Alternatively, chemically controlled glucose-responsive systems have been widely investigated during the last few decades. ${ }^{1,2,7}$ Typically, the insulin embedded matrix with glucose-responsive elements can adjust the insulin release rate through structural changes such as swelling, shrinking, degradation, or dissociation in response to ambient glucose levels. ${ }^{9-11}$ The common glucoseresponsive elements include glucose oxidase (GOx), ${ }^{10-15}$ phenylboronic (PBA), ${ }^{16,17}$ and glucose-binding protein (GBP). ${ }^{18}$ Despite these available chemistries, few synthetic glucose-responsive systems show promise in vivo. Challenges remain to demonstrate a system consisting of fast responsiveness with kinetics similar to a healthy pancreas, biocompatibility without long-term side effects, and ease of administration.

$\mathrm{GOx}$ is an enzyme that can catalyze the oxidation of glucose in the presence of oxygen

$$
\text { glucose }+\mathrm{O}_{2}+\mathrm{H}_{2} \mathrm{O} \stackrel{\text { GOx }}{\longrightarrow} \text { gluconic acid }+\mathrm{H}_{2} \mathrm{O}_{2}
$$

Received: September 13, 2016

Revised: December 24, 2016

Published: January 12, 2017 

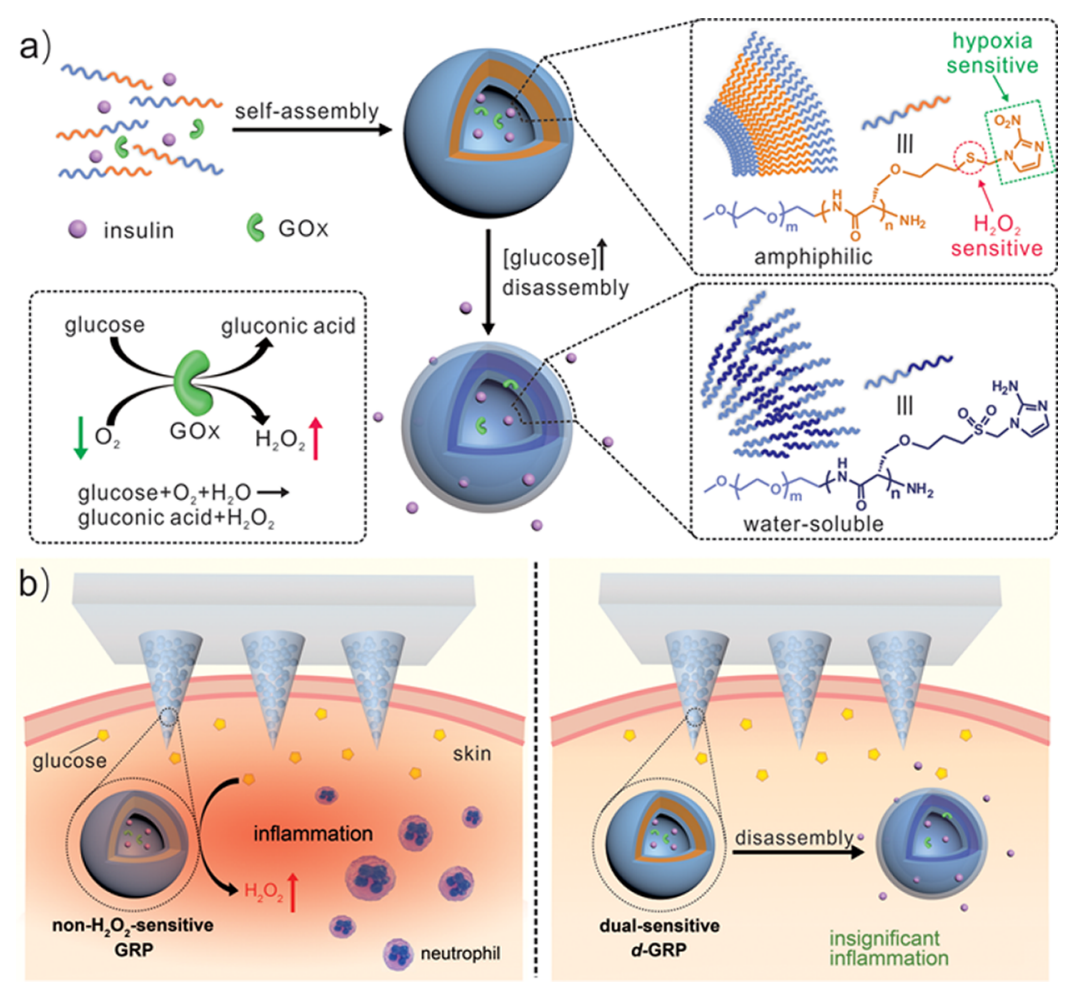

Figure 1. Schematic of the glucose-responsive insulin delivery system using hypoxia and $\mathrm{H}_{2} \mathrm{O}_{2}$ dual-sensitive polymersome-based vesicles ( $d$-GRPs) loading microneedle-array patches. (a) Formation and mechanism of $d$-GRPs comprised of PEG-poly(Ser-S-NI). (b) Schematic of local inflammation induced by non- $\mathrm{H}_{2} \mathrm{O}_{2}$-senstive GRP-loaded microneedle-array patch, and schematic of $d$-GRP-loaded microneedle-array patch for in vivo insulin delivery triggered by a hyperglycemic state for potential prevention of the long-term side effect associated with inflammation.

Previous glucose-responsive systems using GOx typically employed a $\mathrm{pH}$-sensitive matrix with entrapped or immobilized GOx to cause a local decline of $\mathrm{pH}$ with increasing glucose levels, which promoted insulin release through either degradation or protonation. ${ }^{10,11,15,19}$ Recently, our group reported a hypoxia-sensitive mechanism for achieving fast, glucose-responsive, insulin delivery. ${ }^{20} \mathrm{We}$ also demonstrated that utilizing the rapid oxygen consumption during the oxidation of glucose catalyzed by $\mathrm{GOx}$ as a trigger was able to achieve fast insulin release under physiological conditions.

Herein, we report a new hypoxia and $\mathrm{H}_{2} \mathrm{O}_{2}$ dual-sensitive diblock copolymer consisting of poly(ethylene glycol) (PEG) and polyserine modified with 2-nitroimidazole via a thioether moiety (designated PEG-poly(Ser-S-NI); Figure S1). The thioether serves as a $\mathrm{H}_{2} \mathrm{O}_{2}$-sensitive moiety that turns the polymer more hydrophilic when it is converted into a sulfone by $\mathrm{H}_{2} \mathrm{O}_{2}{ }^{21-23}$ This amphiphilic polymer is able to selfassemble into a nanoscaled bilayer vesicle structure (polymersome), which encapsulates recombinant human insulin and $\mathrm{GOx}$ in the aqueous core. When exposed to a high blood glucose level, the glucose diffuses across the polymeric bilayer membrane and interacts with GOx (Figure 1a). During this glucose oxidation process catalyzed by GOx, the dissolved oxygen is rapidly consumed. The resulting local hypoxic environment can promote the bioreduction of NI groups into hydrophilic 2-aminoimidazoles catalyzed by a series of nitroreducatases. $^{20,24,25}$ During the enzymatic oxidation of glucose, the undesirable byproduct $\mathrm{H}_{2} \mathrm{O}_{2}$ is also generated, which may lead to free radical-induced damage to skin tissue during the long-term usage ${ }^{26,27}$ and reduce the activity of GOx, decreasing response rate. ${ }^{28,29}$ Generally, catalase (CAT) is incorporated with GOx-based glucose-responsive systems to scavenge $\mathrm{H}_{2} \mathrm{O}_{2} \cdot{ }^{30}$ However, oxygen regenerates during the decomposition of $\mathrm{H}_{2} \mathrm{O}_{2}$ catalyzed by CAT, which reduces the hypoxic level and leads to low release rate. The $\mathrm{H}_{2} \mathrm{O}_{2}$-sensitive thioether moiety in PEG-poly(Ser-S-NI) has the capability of effectively eliminating the undesirable $\mathrm{H}_{2} \mathrm{O}_{2}$ to assist GOx's catalysis. Meanwhile, the resulting sulfone groups convert the polymer to a more water-soluble form. This change in chemical structure promotes the dissociation of the dual-sensitive, glucose-responsive polymersomes ( $d$-GRPs) and subsequent release of the encapsulated insulin.

In order to achieve convenient administration, the $d$-GRPs can be further integrated with a painless microneedle (MN) patch platform for insulin delivery. ${ }^{20,31-33}$ Once exposed to the high interstitial fluid glucose after transcutaneous administration, the $d$-GRPs loaded in MNs quickly disassociate to release the entrapped insulin into the vascular and lymph capillary network ${ }^{20,34}$ (Figure 1b). Furthermore, unlike non$\mathrm{H}_{2} \mathrm{O}_{2}$-sensitive GRP-loaded $\mathrm{MNs}$, which may lead to a local inflammation due to the rapid accumulation of $\mathrm{H}_{2} \mathrm{O}_{2}$, this "smart insulin patch" (SIP) with a novel hypoxia and $\mathrm{H}_{2} \mathrm{O}_{2}$ dual-sensitive mechanism presents a promising approach for tight glucose regulation and potential prevention of long-term disease morbidity.

The diblock copolymer (PEG-polyserine) was first synthesized via amine-initiated ring-opening. ${ }^{19}$ The (2-nitroimidazol1-yl)methanethiol, which rendered the polymer sensitive to hypoxia and $\mathrm{H}_{2} \mathrm{O}_{2}$ (Figure $\mathrm{S} 1$ ), was conjugated to the hydroxyl group of the serine residue through an allyl ether (PEGpoly(Ser-S-NI)). For comparison purposes, we also synthesized PEG-poly(Ser-NI) without the sulfonate by incorporating the hydroxyl groups of PEG-polyserine with 1-(5-chloropentyl)-2nitroimidazole (Figure S1), which is only able to respond to 
a)

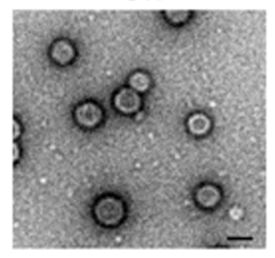

$20 \mathrm{~min}$

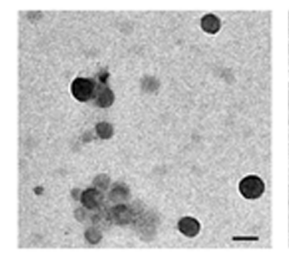

$1 \mathrm{~h}$

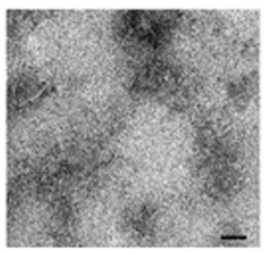

$24 \mathrm{~h}$

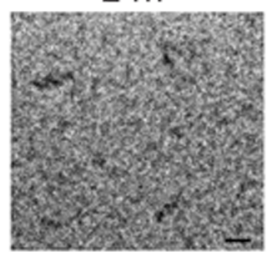

d)
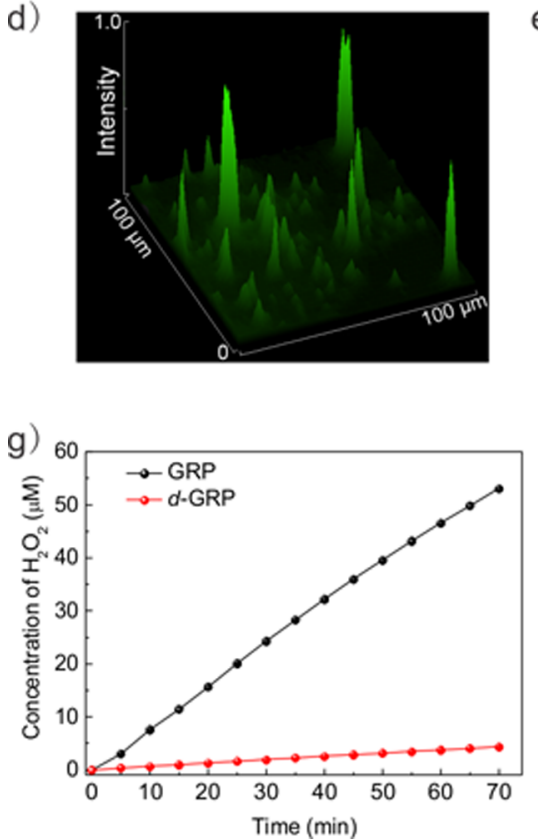

e)

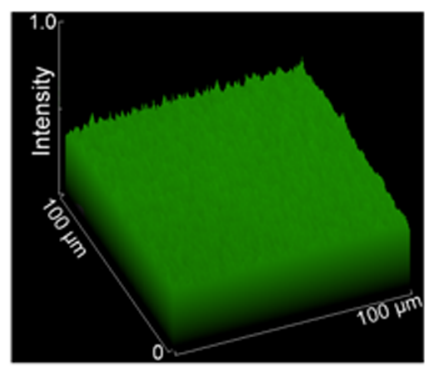

h)

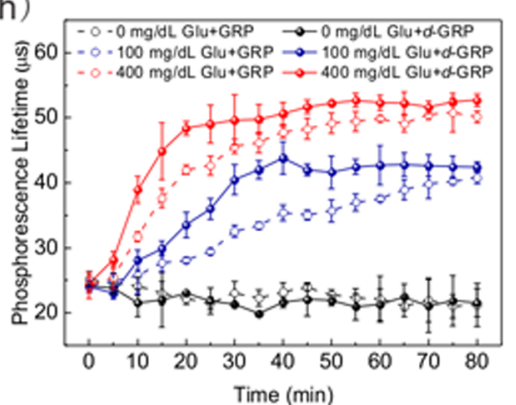

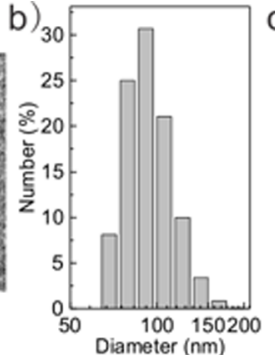

f)

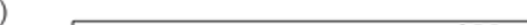

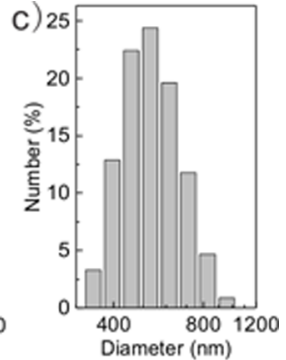

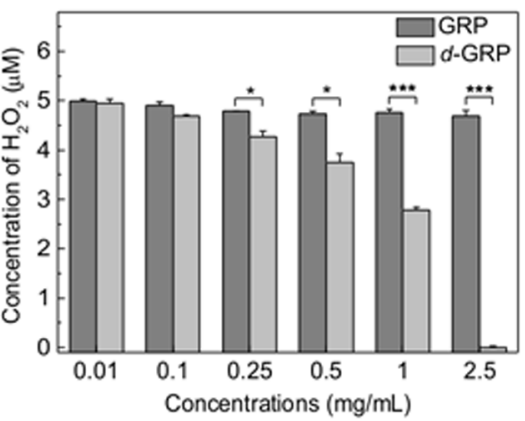

i)

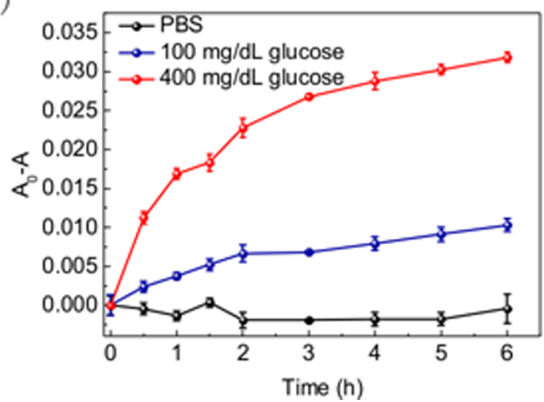

Figure 2. Characterization of dual-sensitive polymersome-based vesicles ( $d$-GRPs). (a) TEM images of $d$-GRPs encapsulating insulin and enzyme pre- or postincubation with $400 \mathrm{mg} / \mathrm{dL}$ glucose for $20 \mathrm{~min}, 1 \mathrm{~h}$, and $24 \mathrm{~h}$. Scale bar is $100 \mathrm{~nm}$. (b,c) Size distribution of $d$-GRPs pre- (b) and post(c) incubation with $400 \mathrm{mg} / \mathrm{dL}$ glucose for $24 \mathrm{~h}$. (d,e) $2.5 \mathrm{D}$ fluorescence images of FITC-insulin loaded $d$-GRPs solution (d) pre- and post- (e) incubated in $400 \mathrm{mg} / \mathrm{dL}$ glucose solution for $24 \mathrm{~h}$ at $37^{\circ} \mathrm{C}$. (f) The sensitivity of $d$-GRPs and GRPs to $\mathrm{H}_{2} \mathrm{O}_{2}$ assessed by a fluormetric hydrogen peroxide assay kit. Student's $t$ test: $* p<0.05$, ***p $<0.001$. (g) The $\mathrm{H}_{2} \mathrm{O}_{2}$ generation rate of GOx-loaded $d$-GRPs or GOx-loaded GRPs incubated in $400 \mathrm{mg} / \mathrm{dL}$ glucose solution. (h) Phosphorescence lifetime profile for the GOx-loaded d-GRPs or GOx-loaded GRPs incubated with different glucose concentration solutions containing an oxygen concentration molecule probe. (i) Decrease of UV absorption at $330 \mathrm{~nm}$ of $d$-GRPs in different glucose concentrations at $37^{\circ} \mathrm{C}$. Error bars indicate $\mathrm{SD}(n=3)$.

hypoxia. PEG-poly(Ser-NI) and serves as a control to confirm the importance of the $\mathrm{H}_{2} \mathrm{O}_{2}$ elimination ability of PEGpoly(Ser-S-NI).

The $d$-GRPs with encapsulated cargoes were formed by selfassembly of PEG-poly(Ser-S-NI) through a solvent evaporation method. ${ }^{1,19}$ As shown in Figure 2a, the transmission electron microscopy (TEM) image showed that the $d$-GRPs had a spherical structure, and a bilayer membrane with a thickness of around $20 \mathrm{~nm}$ was clearly observed. The average diameter was measured as $94 \mathrm{~nm}$ by dynamic light scattering (DLS) (Figure 2b). The successful encapsulation of insulin was further confirmed by fluorescence microscopy imaging of the $d$-GRPs with FITC-labeled insulin (Figure 2d). The insulin loading capacity of $d$-GRPs was determined as $3.2 \%$. Meanwhile, non$\mathrm{H}_{2} \mathrm{O}_{2}$ sensitive GRPs entrapping insulin and GOx were also prepared from PEG-poly(Ser-NI) with the similar morphology and size as a control (Figure S2).

The sensitivity of $d$-GRPs and GRPs to $\mathrm{H}_{2} \mathrm{O}_{2}$ was first assessed by measuring the concentration of $\mathrm{H}_{2} \mathrm{O}_{2}$ using a fluorimetric assay kit for hydrogen peroxide. The initial $\mathrm{H}_{2} \mathrm{O}_{2}$ solution $(5 \mu \mathrm{M})$ showed high emission intensity after reaction with a peroxidase substrate (Figure $2 \mathrm{f}$ ). However, addition of $d$ -
GRPs into the $\mathrm{H}_{2} \mathrm{O}_{2}$ solution produced a significant decline in fluorescence intensity. Furthermore, the reduction in intensity was dependent on the $d$-GRP concentration, while no significant change in the concentration of $\mathrm{H}_{2} \mathrm{O}_{2}$ occurred after incubation with GRP controls. Even more importantly, the $\mathrm{H}_{2} \mathrm{O}_{2}$ elimination capability was assessed through incubation of GOx-loaded vesicles with glucose. As shown in Figure $2 \mathrm{~g}, \mathrm{H}_{2} \mathrm{O}_{2}$ generated rapidly due to the oxidation of glucose when incubating GRPs in PBS buffer [ $137 \mathrm{mM} \mathrm{NaCl}, 2.7 \mathrm{mM} \mathrm{KCl}$, $10 \mathrm{mM} \mathrm{Na} 2 \mathrm{HPO}_{4}, 2 \mathrm{mM} \mathrm{KH}_{2} \mathrm{PO}_{4}$ (pH 7.4)] consisting of 400 $\mathrm{mg} / \mathrm{dL}$ glucose, while the produced $\mathrm{H}_{2} \mathrm{O}_{2}$ was almost eliminated by $d$-GRPs.

In order to evaluate the glucose-responsive capability of $d$ GRPs, vesicles were incubated with PBS buffer containing various concentrations of glucose, including a typical hyperglycemic level $(400 \mathrm{mg} / \mathrm{dL})$, a normoglycemia level $(100 \mathrm{mg} /$ $\mathrm{dL})$, and a control level $(0 \mathrm{mg} / \mathrm{dL})$. In the presence of high glucose level, the dissolved oxygen was rapidly consumed due to the oxidation of glucose catalyzed by GOx. Using an oxygensensitive phosphorescent molecular probe, ${ }^{35}$ a relatively lower oxygen level was generated in the $d$-GRPs under the hyperglycemia level compared to the other two control samples 

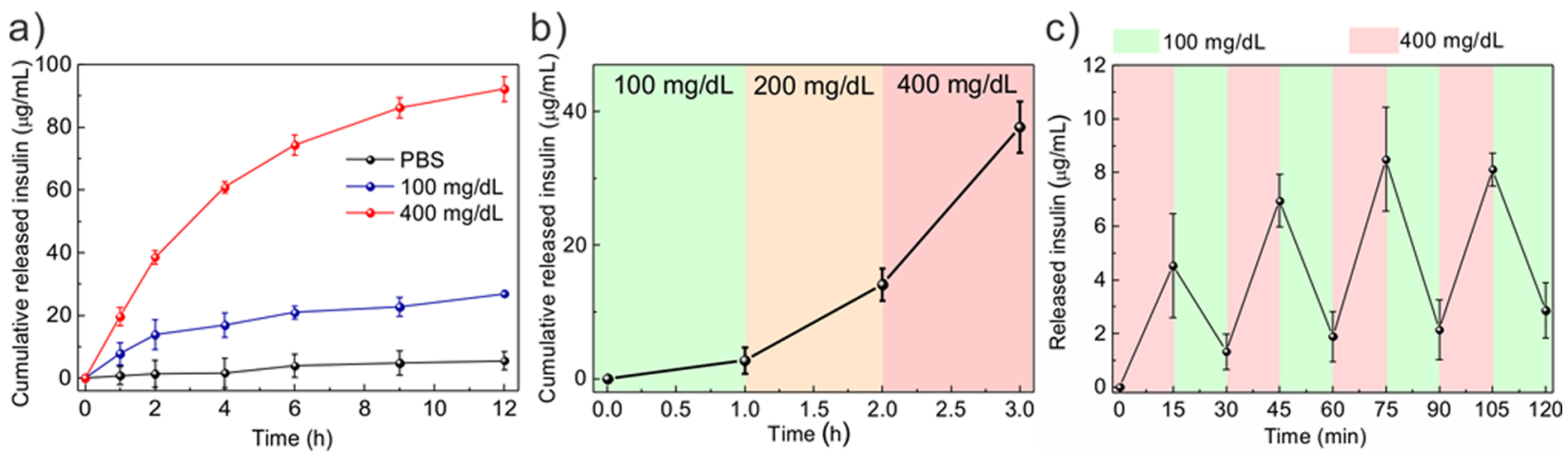

Figure 3. In vitro glucose-responsive release of insulin from $d$-GRPs. (a) In vitro accumulated insulin released from the $d$-GRPs in several glucose concentrations at $37^{\circ} \mathrm{C}$. (b) Self-regulated profiles of the $d$-GRPs present the rate of insulin release as a function of glucose concentration. (c) Pulsatile release profile of $d$-GRPs presents the rate of insulin release as a function of glucose concentrations (100 and $400 \mathrm{mg} / \mathrm{dL}$ ). Error bars indicate $\mathrm{SD}(n=3)$.

(Figure 2h). The oxygen concentration rapidly decreased within the vesicles and reached equilibrium within $20 \mathrm{~min}$. Moreover, a significant slower oxygen consumption rate and higher oxygen level was observed when incubating GRPs with the same concentration in glucose solution. The lower oxygen level in $d$-GRPs sample can be attributed to the effective $\mathrm{H}_{2} \mathrm{O}_{2}$ elimination ability of PEG-poly(Ser-S-NI), which avoids the deactivation of GOx. Under such hypoxic condition, the NI groups were effectively reduced by NADPH catalyzed by the reductase. $^{20,24,36}$ Correspondingly, the characteristic absorbance of NI at $330 \mathrm{~nm}$ gradually declined over time, ${ }^{37}$ confirming the conversion of hydrophobic NI groups to hydrophilic 2aminoimidazoles groups (Figure 2i). Because of the generation of hydrophilic 2-aminoimidazoles and sulfone on PEGpoly(Ser-S-NI), the $d$-GRPs began to dissociate and subsequently released the encapsulated cargoes. The corresponding change in morphology and size were clearly observed by transmission electron microscopy and dynamic light scattering (Figure 2a,c). Furthermore, the release of FITC-labeled insulin was validated using fluorescence microscopy. As shown in the 2.5D fluorescence images, the $d$-GRPs contain less insulin and a present homogeneous distribution after incubation with 400 $\mathrm{mg} / \mathrm{dL}$ glucose solution, confirming the glucose-triggered insulin release (Figure 2e).

The quick release of insulin was obtained after exposure to a high glucose solution due to the dissociation of $d$-GRPs, while only a small amount of released insulin was observed in the control samples under 0 or $100 \mathrm{mg} / \mathrm{dL}$ glucose levels (Figure $3 \mathrm{a}, \mathrm{b})$. Furthermore, a pulsatile release profile of insulin was achieved when $d$-GRPs were alternatively immersed in the normal and hyperglycemic solutions for several cycles (Figure $3 c)$. The release rates changed in response to the change of glucose levels, indicating the disassociation of $d$-GRPs, and the amount of insulin released was dependent on the glucose concentration. The insulin release rate could also be varied by altering the GOx concentration. A maximum of an 11.2-fold difference in insulin release rate was observed after incubation under 100 or $400 \mathrm{mg} / \mathrm{dL}$ glucose levels for $2 \mathrm{~h}$ when reducing the weight ratio of GOx to insulin to 1:40 (Figure S3). Additionally, the insulin itself was not denatured during the encapsulation or release procedures; the secondary conformational structure, measured using circular dichroism, of released insulin from $d$-GRPs did not change compared to that of the native insulin (Figure S4).
In order to achieve convenient and painless administration, ${ }^{20,38} d$-GRPs were integrated with a cross-linked MN-array patch composed of cross-linked hyaluronic acid using a micromolding approach. The resulting MNs were arranged in a $20 \times 20$ array, and each needle was of conical shape with diameters of $300 \mu \mathrm{m}$ at the base and $10 \mu \mathrm{m}$ at the tip and a height of $600 \mu \mathrm{m}$ (Figure $4 \mathrm{a}, \mathrm{b}$ ). The fluorescence image in Figure $4 \mathrm{c}$ displays a representative rhodamine-labeled $\mathrm{MN}$ with FITC-insulin-loaded $d$-GRPs, indicating $d$-GRPs were well distributed in the tip region of each needle. The mechanical strength of the MN was measured as $3 \mathrm{~N} /$ needle using a tensile compression machine (Figure S5), which was sufficient for skin insertion without breaking. ${ }^{31}$

We next evaluated the in vivo efficacy of the SIP for diabetes treatment using streptozotocin-induced adult type 1 diabetic C57BL/6J mice. The diabetic mice were randomly divided into four groups $(n=5)$ and the following patches transcutaneously attached: the empty MNs containing only cross-linked HA, MNs loaded with human recombinant insulin, MNs loaded with $d$-GRPs encapsulating GOx and insulin $[d-G R P(E+I)]$, and MNs loaded with $d$-GRPs encapsulating insulin only $[d-$ GRP(I)] (insulin dose: $10 \mathrm{mg} / \mathrm{kg}$ ). The trypan blue staining of needle penetration sites was clearly observed on the excised skin sample, and the hematoxylin and eosin (H\&E)-stained slide further verified that MNs could easily penetrate to the epidermis (Figure S6), which exposed the d-GRPs to the interstitial fluid. After removal of the MNs, the punctures in the skin rapidly disappeared and were not evident at $4 \mathrm{~h}$ (Figure S7).

The BGLs of each group were closely monitored after administration. As shown in Figure 4d, a rapid decline of BGLs was observed in the group treated with $d$-GRP $(\mathrm{E}+\mathrm{I})$-loaded MNs in the first $1 \mathrm{~h}$, and the BGLs maintained in a normal state for up to $6 \mathrm{~h}$ without peaks of hypoglycemia. In contrast, without the enzyme GOx, the BGLs of mice treated with $d$ GRP(I)-loaded MNs did not show an obvious decrease, indicating that $d$-GRPs were highly stable in normal tissues. Correspondingly, the plasma human insulin levels in mice treated with $d-G R P(E+I)$-loaded MNs were higher than those treated with $d$-GRP(I)-loaded $\mathrm{MNs}$ for at least $24 \mathrm{~h}$ (Figure $4 \mathrm{e})$.

To further investigate the in vivo glucose control capability of $\mathrm{MNs}$, an additional administration with MNs was performed $2.5 \mathrm{~h}$ post the first administration. Unlike the group administered with free insulin-loaded MNs, the BGLs of the 
a)

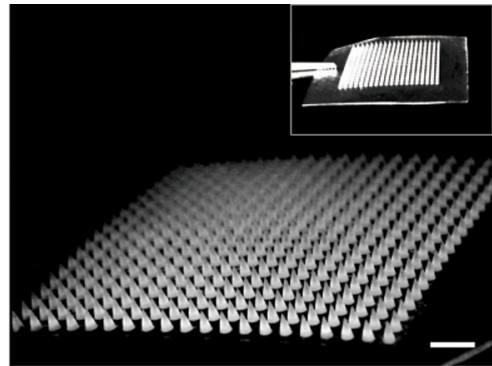

d)

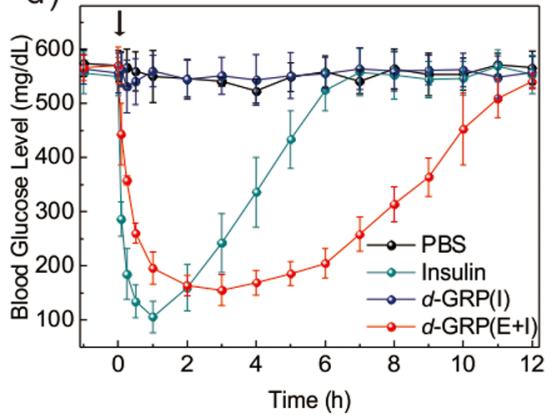

b)

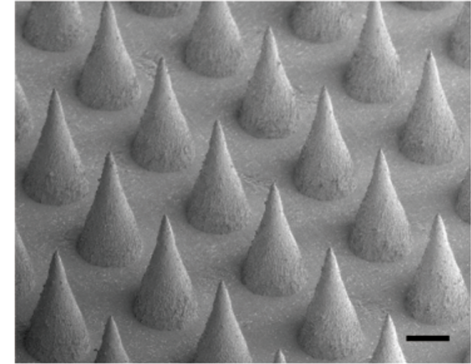

e)

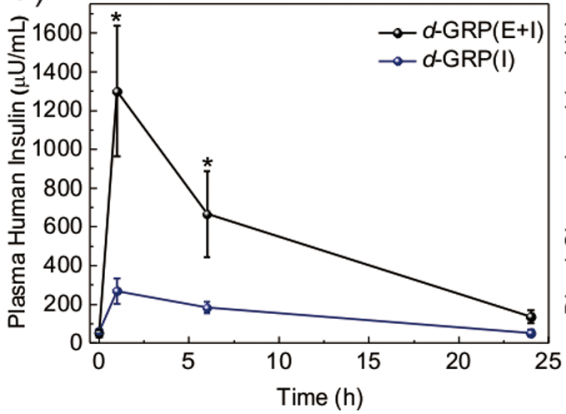

c)

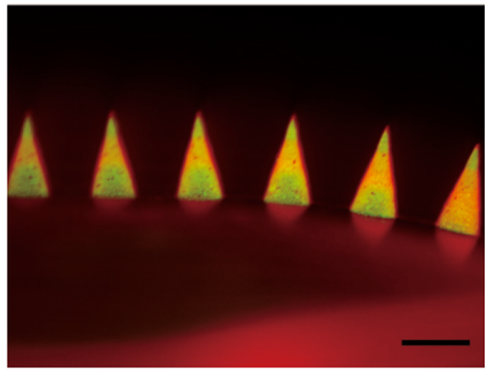

f)

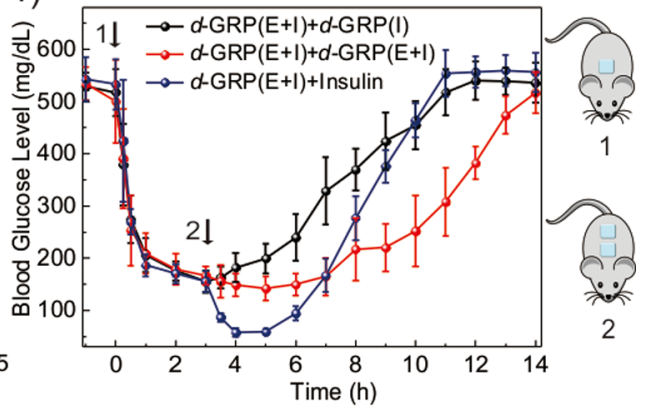

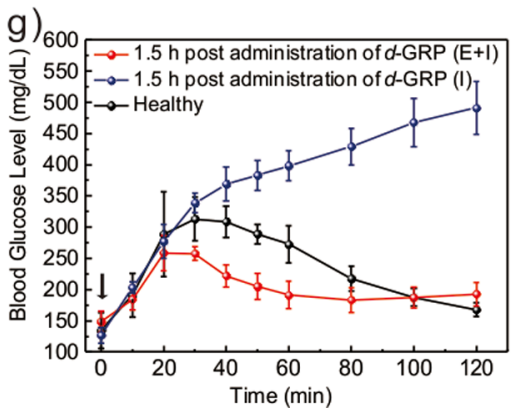
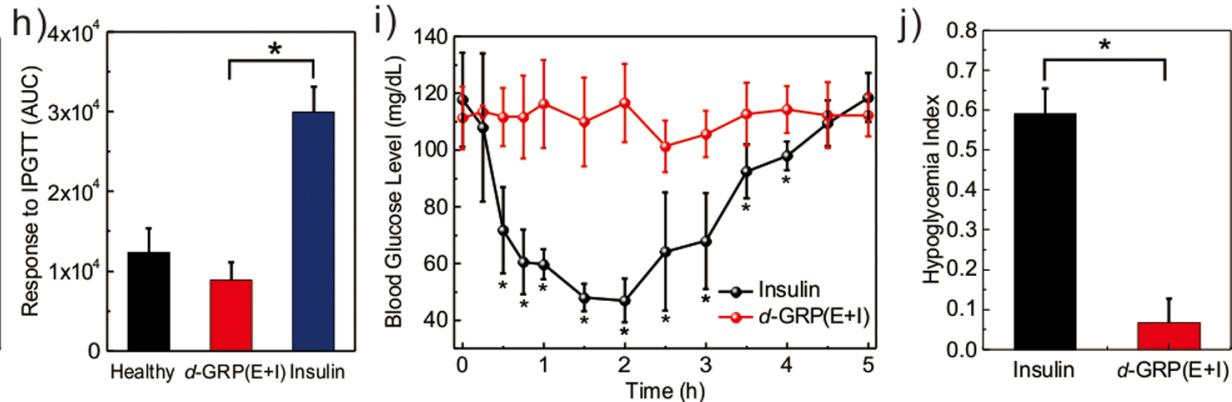

Figure 4. In vivo studies of the MN-array patches for type 1 diabetes treatment. (a) Photos of MNs array. Scale bars are $1 \mathrm{~mm}$. (b) A scanning electron microscopy image of MNs array. Scale bar is $200 \mu \mathrm{m}$. (c) A fluorescence microscopy image of rhodamine-labeled MNs loading $d$-GRPs with FITC-labeled insulin. Scale bar is $500 \mu \mathrm{m}$. (d,e) In vivo studies of the $\mathrm{MN}$-array patches for type 1 diabetes treatment: blood glucose levels (d) and plasma human insulin concentrations (e) in streptozotocin-induced diabetic mice after treatment with blank MNs containing only cross-linked HA, MNs loaded with human recombinant insulin, MNs loaded with $d$-GRPs containing insulin and enzyme ( $d$-GRP(E+I)), or MNs loaded with $d$-GRPs containing insulin $(d-\mathrm{GRP}(\mathrm{I}))$. ${ }^{*} p<0.05$ for administration with $d$-GRP(E+I)-loaded MNs compared with administration of $d$-GRP(I). (f) The blood glucose changes of mice treated with additional administration with $\mathrm{MN}$-array patch $1 \mathrm{~h}$ post administration of $d$-GRP(E+I)-loaded $\mathrm{MNs}$. $(\mathrm{g})$ In vivo glucose tolerance test toward diabetic mice $1.5 \mathrm{~h}$ post administration of $d$-GRP(E+I)-loaded MNs or insulin-loaded MNs in comparison with the healthy control mice. (h) The responsiveness was calculated based on the area under the curve in 120 min with the baseline set at the 0 min blood glucose reading. $* p<0.05$ for administration with $d$-GRP(E+I)-loaded MNs compared with administration of insulin-loaded MNs. (i) The blood glucose changes of healthy mice administrated with $\mathrm{MN}$-array patch over time. ${ }^{*} p<0.05$ for administration with $d$-GRP(E+I)-loaded MNs compared with insulin-loaded MNs. (j) Quantification of hypoglycemia index, which was calculated from the difference between the initial and nadir blood glucose readings divided by the time at which nadir was reached. ${ }^{*} p<0.05$ for administration with $d$-GRP(E+I)-loaded MNs compared with insulin-loaded MNs. The black arrows indicate the administration points. Error bars indicate SD $(n=5)$.

mice administered with $d$-GRP(E+I)-loaded $\mathrm{MNs}$ did not further decline to a hyperglycemic state (Figure 4f), supporting the idea that these smart insulin patches can efficiently avoid a risk of hypoglycemia. More importantly, the application of an additional patch was able to significantly prolong the treatment efficacy in response to the elevated BGLs compared to one patch.

Next, an intraperitoneal glucose tolerance test (IPGTT) ${ }^{17}$ was performed at $1.5 \mathrm{~h}$ after administration of MNs in order to further evaluate the dynamic of insulin release in vivo. The BGLs of diabetic mice treated with $d$-GRP(E+I)-loaded MNs showed a relatively delayed increase after glucose injection and then rapidly declined to a normoglycemic state within $60 \mathrm{~min}$ (Figure $4 \mathrm{~g}$ ). In sharp contrast, the mice treated with insulinloaded MNs showed a gradual increase in blood glucose in 120 $\min$. To quantitate the glucose response to the various MNs, the area under the curve was calculated between 0 and $120 \mathrm{~min}$ for each group. As shown, the $d-\mathrm{GRP}(\mathrm{E}+\mathrm{I})$-loaded administered mice showed significantly enhanced glucose responsiveness to the glucose challenge (Figure $4 \mathrm{~h}$ ).

To examine the potential for induction of hypoglycemia by MNs further, we studied their effect on the healthy mice. As shown in Figure 4i, the insulin-loaded $M N s$ produced remarkably reduced BGLs compared to $d-G R P(E+I)$-loaded MNs-treated mice, indicating that there was little insulin leak in $d$-GRP-loaded MNs. The corresponding hypoglycemia index was calculated to measure the risk of hypoglycemia. $d$-GRPsloaded MNs exhibited a remarkable reduced hypoglycemia index compared to the free insulin-loaded MNs (Figure 4j).

To study the biocompatibility of MNs loaded with $d$-GRPs, both $d$-GRPs-loaded MNs and GRPs-loaded MNs without $\mathrm{H}_{2} \mathrm{O}_{2}$ eliminating ability were transcutaneously attached to a 
a)

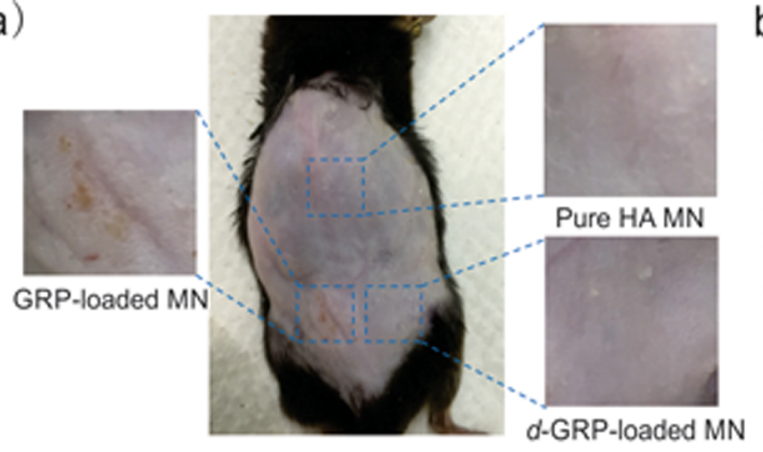

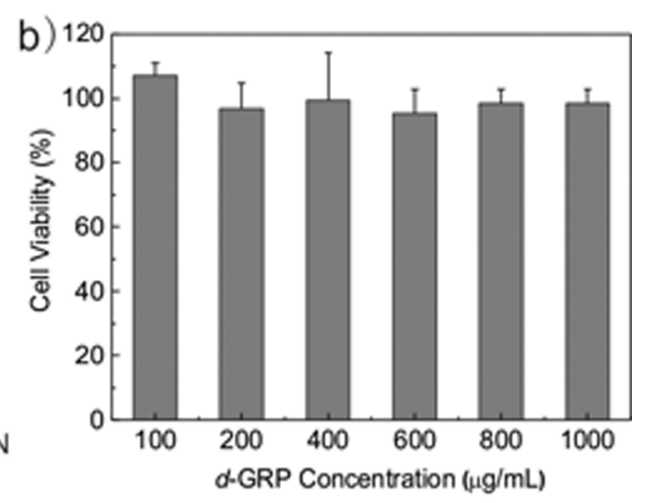

c)
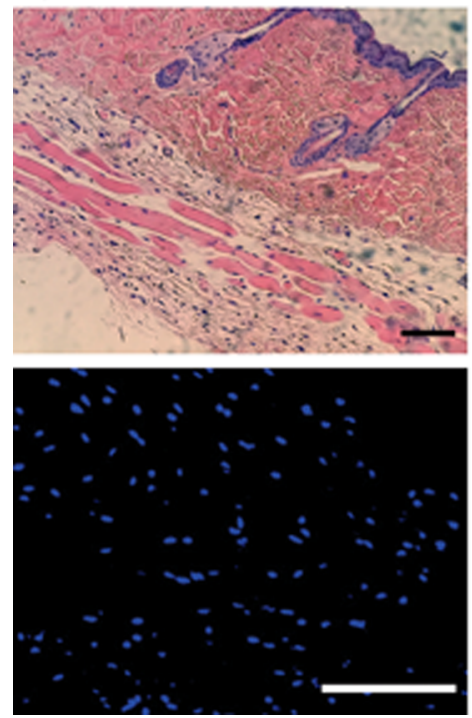
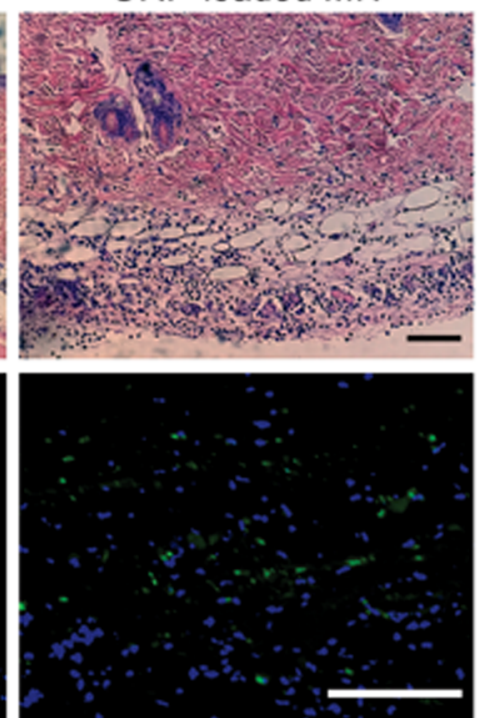

GRP-loaded MN
$d$-GRP-loaded MN
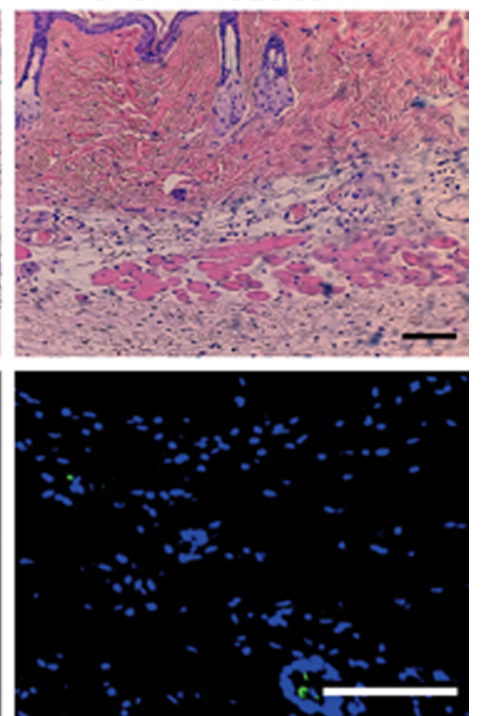

Figure 5. Biocompatibility evaluation of $d$-GRP-loaded MNs. (a) Photograph of a mouse transcutaneously administrated with pure hyaluronic acid (HA) MNs, GRP-loaded MNs, and $d$-GRP-loaded MNs at different sites. (b) Cytotoxicity study of bare $d$-GRPs after 24 h incubation with HeLa cells. Error bars indicate SD $(n=6)$. (c) Micrographs of mouse skin tissue at the treated sites. Skin tissue was collected from the mouse shown in panel a. Top: H\&E stained images. Bottom: Histology stain with TUNEL assay (green) and Hoechst (blue). Scale bar: $100 \mu \mathrm{m}$.

single mouse at different sites. Meanwhile, the pure hyaluronic acid MNs were attached to the same mouse as a negative control. Under a high GOx dose $(3 \mathrm{mg} / \mathrm{kg}$ ), obvious skin damage was observed at the site where GRP(E)-loaded MNs had been attached for 2 days, while there were no significant lesions at the sites treated with $d$-GRP(E)-loaded $\mathrm{MNs}$ or pure hyaluronic acid MNs (Figure 5a). The histological images using $\mathrm{H} \& \mathrm{E}$ staining showed that $\mathrm{GRP}(\mathrm{E})$-loaded $\mathrm{MNs}$ caused neutrophil infiltration (Figure $5 \mathrm{c}$ top), indicating a pathophysiological response and tissue damage induced by the generated $\mathrm{H}_{2} \mathrm{O}_{2}$. In contrast, no significant pathological abnormalities occurred in the site treated with $d$-GRP(E)-loaded MNs. Moreover, the skin tissue stained with the in situ terminal deoxyribonucleotidyl transferase (TDT)-mediated dUTP-digoxigenin nick end labeling (TUNEL) assay clearly showed the cell apoptosis in the skin sample treated with GRP(E)-loaded MNs, whereas no cell death in the skin tissue treated with the $d$-GRP(E)-loaded MNs and pure hyaluronic acid MNs (Figure $5 \mathrm{c}$ bottom). Furthermore, insignificant pathological abnormality was observed in skin tissue after a 2 week treatment of $d$ GRP(E+I)-loaded MNs with a normal GOx dose of $1 \mathrm{mg} / \mathrm{kg}$ (Figure S8). A long-term safety assessment will be further performed on diabetic minipigs, the skin of which has more similar physical and physiological properties to that of human beings. ${ }^{39}$ The cytotoxicity of bare $d$-GRPs toward HeLa cells was evaluated by 3-(4,5)-dimethylthiahiazo(-z-y1)-3,5-diphenytetrazoliumromide (MTT) assay. As presented in Figure 5b, the bare $d$-GRPs did not show significant toxicity within all the studied concentrations.

In conclusion, an effective glucose-responsive insulin delivery strategy has been exploited utilizing vesicles sensitive to both hypoxia and $\mathrm{H}_{2} \mathrm{O}_{2}$. A local hypoxic environment can be quickly generated due to the oxygen consumption during the enzymatic conversion of glucose to gluconic acid, which facilitates the solubility switch of the polymer through the bioreduction of NI groups on the side chains. Moreover, the thioether moiety within the designed polymer not only responds to $\mathrm{H}_{2} \mathrm{O}_{2}$, the byproduct during glucose oxidation, to promote the disassembly of vesicles, but also eliminates the excess $\mathrm{H}_{2} \mathrm{O}_{2}$ to maintain the activity of GOx and circumvent the damage to skin tissue. Furthermore, the $d$-GRPs can be integrated within a cross-linked HA-based $\mathrm{MN}$-array patch to achieve convenient, painless, and continuous administration of insulin. The in vivo studies demonstrated that this SIP was highly effective in tight regulation of BGLs in diabetic mice and showed minimal side effects regarding inflammation. Additionally, this dual-sensitive formulation strategy displays the potential benefit in controlled delivery for other therapeutic agents under hypoxia and high oxidative stress. ${ }^{40}$ For potential translation of this microneedle array patch-based formulation, efforts associated with further 
enhancement of loading capability and bioavailability are expected. In addition, by tuning physicochemical property of major material as well as formulation composites, optimization of glucose-responsive pharmacokinetics in order to mimic that of healthy pancreatic cells is also essential. Thorough characterization utilizing animals equipped with the continuous glucose monitoring systems (CGMSs) would facilitate to obtain detailed information to guide the improvement.

\section{ASSOCIATED CONTENT}

\section{S Supporting Information}

The Supporting Information is available free of charge on the ACS Publications website at DOI: 10.1021/acs.nanolett.6b03848.

Materials and methods and supplementary Figures S1S8 (PDF)

\section{AUTHOR INFORMATION}

\section{Corresponding Author}

*E-mail: zgu@email.unc.edu. Phone: 1-919-515-7944.

\section{ORCID}

Zhen Gu: 0000-0003-2947-4456

\section{Author Contributions}

The manuscript was written through contributions of all authors. All authors have given approval to the final version of the manuscript. J.Y. and C.Q. contributed equally.

\section{Notes}

The authors declare no competing financial interest.

\section{ACKNOWLEDGMENTS}

This work was supported by the grants from JDRF (Grant 3SRA-2015-117-Q-R), and NC TraCS, NIH's Clinical and Translational Science Awards (CTSA, NIH 1UL1TR001111) at $\mathrm{UNC}-\mathrm{CH}$. We acknowledge the use of the Analytical Instrumentation Facility (AIF) at NC State, which is supported by the State of North Carolina and the National Science Foundation (NSF).

\section{REFERENCES}

(1) Mo, R.; Jiang, T.; Di, J.; Tai, W.; Gu, Z. Chem. Soc. Rev. 2014, 43, 3595-3629.

(2) Veiseh, O.; Tang, B. C.; Whitehead, K. A.; Anderson, D. G.; Langer, R. Nat. Rev. Drug Discovery 2014, 14, 45-57.

(3) Owens, D. R.; Zinman, B.; Bolli, G. B. Lancet 2001, 358, 739746.

(4) Bratlie, K. M.; York, R. L.; Invernale, M. A.; Langer, R.; Anderson, D. G. Adv. Healthcare Mater. 2012, 1, 267-284.

(5) Ohkubo, Y.; Kishikawa, H.; Araki, E.; Miyata, T.; Isami, S.; Motoyoshi, S.; Kojima, Y.; Furuyoshi, N.; Shichiri, M. Diabetes Res. Clin. Pract. 1995, 28, 103-117.

(6) Wu, Q.; Wang, L.; Yu, H.; Wang, J.; Chen, Z. Chem. Rev. 2011, $111,7855-7875$.

(7) Gilroy, C. A.; Luginbuhl, K. M.; Chilkoti, A. J. Controlled Release 2016, 240, 151-164.

(8) Pickup, J. C. N. Engl. J. Med. 2012, 366, 1616-1624.

(9) Gordijo, C. R.; Koulajian, K.; Shuhendler, A. J.; Bonifacio, L. D.; Huang, H. Y.; Chiang, S.; Ozin, G. A.; Giacca, A.; Wu, X. Y. Adv. Funct. Mater. 2011, 21, 73-82.

(10) Gu, Z.; Dang, T. T.; Ma, M.; Tang, B. C.; Cheng, H.; Jiang, S.; Dong, Y.; Zhang, Y.; Anderson, D. G. ACS Nano 2013, 7, 6758-6766.

(11) Gu, Z.; Aimetti, A. A.; Wang, Q.; Dang, T. T.; Zhang, Y.; Veiseh, O.; Cheng, H.; Langer, R. S.; Anderson, D. G. ACS Nano 2013, 7, 4194-4201.
(12) Fischel-Ghodsian, F.; Brown, L.; Mathiowitz, E.; Brandenburg, D.; Langer, R. Proc. Natl. Acad. Sci. U. S. A. 1988, 85, 2403-2406.

(13) Kang, S. I.; Bae, Y. H. J. Controlled Release 2003, 86, 115-121.

(14) Podual, K.; Doyle, F.; Peppas, N. Polymer 2000, 41, 3975-3983.

(15) Podual, K.; Doyle, F. J.; Peppas, N. A. J. Controlled Release 2000, 67, 9-17.

(16) Kataoka, K.; Miyazaki, H.; Bunya, M.; Okano, T.; Sakurai, Y. J. Am. Chem. Soc. 1998, 120, 12694-12695.

(17) Chou, D. H.-C.; Webber, M. J.; Tang, B. C.; Lin, A. B.; Thapa, L. S.; Deng, D.; Truong, J. V.; Cortinas, A. B.; Langer, R.; Anderson, D. G. Proc. Natl. Acad. Sci. U. S. A. 2015, 112, 2401-2406.

(18) Makino, K.; Mack, E. J.; Okano, T.; Kim, S. W. J. Controlled Release 1990, 12, 235-239.

(19) Tai, W.; Mo, R.; Di, J.; Subramanian, V.; Gu, X.; Buse, J. B.; Gu, Z. Biomacromolecules 2014, 15, 3495-3502.

(20) Yu, J.; Zhang, Y.; Ye, Y.; DiSanto, R.; Sun, W.; Ranson, D.; Ligler, F.; Buse, J.; Gu, Z. Proc. Natl. Acad. Sci. U. S. A. 2015, 112, $8260-8265$.

(21) Napoli, A.; Valentini, M.; Tirelli, N.; Müller, M.; Hubbell, J. A. Nat. Mater. 2004, 3, 183-189.

(22) Huo, M.; Yuan, J.; Tao, L.; Wei, Y. Polym. Chem. 2014, 5, 15191528.

(23) Deng, Z.; Qian, Y.; Yu, Y.; Liu, G.; Hu, J.; Zhang, G.; Liu, S. J. Am. Chem. Soc. 2016, 138, 10452-10466.

(24) Nunn, A.; Linder, K.; Strauss, H. W. Eur. J. Nucl. Med. 1995, 22, 265-280.

(25) Krohn, K. A.; Link, J. M.; Mason, R. P. J. Nucl. Med. 2008, 49, 129S-148S.

(26) Kohen, R. Biomed. Pharmacother. 1999, 53, 181-192.

(27) Liu, Y.; Du, J.; Yan, M.; Lau, M. Y.; Hu, J.; Han, H.; Yang, O. O.; Liang, S.; Wei, W.; Wang, H. Nat. Nanotechnol. 2013, 8, 187-192.

(28) Traitel, T.; Cohen, Y.; Kost, J. Biomaterials 2000, 21, 16791687.

(29) Zhang, K.; Wu, X. Y. J. Controlled Release 2002, 80, 169-178.

(30) Beers, R. F.; Sizer, I. W. J. Biol. Chem. 1952, 195, 133-140.

(31) Prausnitz, M. R. Adv. Drug Delivery Rev. 2004, 56, 581-7.

(32) Yang, S. Y.; O'Cearbhaill, E. D.; Sisk, G. C.; Park, K. M.; Cho, W. K.; Villiger, M.; Bouma, B. E.; Pomahac, B.; Karp, J. M. Nat. Commun. 2013, 4, 1702.

(33) Yang, S.; Wu, F.; Liu, J.; Fan, G.; Welsh, W.; Zhu, H.; Jin, T. Adv. Funct. Mater. 2015, 25, 4633-4641.

(34) Heo, Y. J.; Shibata, H.; Okitsu, T.; Kawanishi, T.; Takeuchi, S. Proc. Natl. Acad. Sci. U. S. A. 2011, 108, 13399-13403.

(35) Will, Y.; Hynes, J.; Ogurtsov, V. I.; Papkovsky, D. B. Nat. Protoc. 2007, 1, 2563-2572.

(36) Takasawa, M.; Moustafa, R. R.; Baron, J.-C. Stroke 2008, 39, 1629-1637.

(37) Seki, Y.; Nakamura, T.; Okami, Y. J. Biochem. 1970, 67, 389396.

(38) Prausnitz, M. R.; Langer, R. Nat. Biotechnol. 2008, 26, 12611268.

(39) Summerfield, A.; Meurens, F.; Ricklin, M. E. Mol. Immunol. 2015, 66, 14-21.

(40) Lu, Y.; Aimetti, A. A.; Langer, R.; Gu, Z. Nat. Rev. Mater. 2016, 1,16075 . 